\title{
Spatially resolving the outer atmosphere of the $M$ giant BK Virginis in the CO first overtone lines with VLTI/AMBER ${ }^{\star}$
}

\author{
K. Ohnaka ${ }^{1}$, K.-H. Hofmann ${ }^{1}$, D. Schertl ${ }^{1}$, G. Weigelt ${ }^{1}$, F. Malbet ${ }^{2}$, F. Massi ${ }^{3}$, A. Meilland ${ }^{4}$, and Ph. Stee ${ }^{4}$ \\ 1 Max-Planck-Institut für Radioastronomie, Auf dem Hügel 69, 53121 Bonn, Germany \\ e-mail: kohnaka@mpifr.de \\ 2 UJF-Grenoble 1/CNRS-INSU, Institut de Planétologie et d'Astrophysique de Grenoble (IPAG) UMR 5274, 38041 Grenoble, France \\ 3 INAF - Osservatorio Astrofisico di Arcetri, Instituto Nazionale di Astrofisica, Largo E. Fermi 5, 50125 Firenze, Italy \\ ${ }^{4}$ Observatoire de la Côte d'Azur, Département FIZEAU, Boulevard de l'Observatoire, BP 4229, 06304 Nice Cedex 4, France
}

Received 20 September 2011 / Accepted 18 November 2011

\begin{abstract}
Context. The mass-loss mechanism in normal $\mathrm{K}-\mathrm{M}$ giant stars with small variability amplitudes is not yet understood, although the majority among red giant stars are precisely of this type.

Aims. We present high-spatial and high-spectral resolution observations of the M7 giant BK Vir with AMBER at the Very Large Telescope Interferometer (VLTI). Our aim is to probe the physical properties of the outer atmosphere by spatially resolving the star in the individual $\mathrm{CO}$ first overtone lines.

Methods. BK Vir was observed between 2.26 and $2.31 \mu \mathrm{m}$ using the 16-32-48 m telescope configuration with an angular resolution of 9.8 mas and a spectral resolution of 12000 .

Results. The uniform-disk diameters observed in the CO first overtone lines are 12-31\% larger than those measured in the continuum. We also detected asymmetry in the CO line-forming region, which manifests itself as non-zero/non- $\pi$ differential and closure phases. The data taken 1.5 months apart show possible time variation on a spatial scale of 30 mas (corresponding to $3 \times$ stellar diameter) at the $\mathrm{CO}$ band head. Comparison of the observed data with the MARCS photospheric model shows that whereas the observed CO line spectrum can be satisfactorily reproduced by the model, the angular sizes observed in the CO lines are much larger than predicted by the model. Our model with two additional CO layers above the MARCS photosphere reproduces the observed spectrum and interferometric data in the CO lines simultaneously. This model suggests that the inner CO layer at $\sim 1.2 R_{\star}$ is very dense and warm with a CO column density of $\sim 10^{22} \mathrm{~cm}^{-2}$ and temperatures of $1900-2100 \mathrm{~K}$, while the outer CO layer at $2.5-3.0 R_{\star}$ is characterized by column densities of $10^{19}-10^{20} \mathrm{~cm}^{-2}$ and temperatures of $1500-2100 \mathrm{~K}$.

Conclusions. Our AMBER observations of BK Vir have spatially resolved the extended molecular outer atmosphere of a normal $\mathrm{M}$ giant in the individual CO lines for the first time. The temperatures derived for the CO layers are higher than, or equal to, the uppermost layer of the MARCS photospheric model, implying the operation of some heating mechanism in the outer atmosphere. This study also illustrates that testing photospheric models only with the spectra of strong molecular or atomic features can be misleading.
\end{abstract}

Key words. stars: AGB and post-AGB - techniques: interferometric - stars: atmospheres - stars: individual: BK Virginis infrared: stars - techniques: high angular resolution

\section{Introduction}

The mass loss in late evolutionary stages of low- and intermediate-mass stars $\left(1 \lesssim M / M_{\odot} \lesssim 8\right)$, particularly in the red giant branch (RGB) and asymptotic giant branch (AGB), is important not only for the evolution of the star itself but also for the chemical enrichment of the interstellar medium. The mass loss in Mira-type stars in the AGB phase, which are characterized by the large-amplitude pulsation with a variability amplitude of $\Delta V \approx 6 \mathrm{mag}$ and a period of $\sim 1$ year, has been observationally and theoretically intensively studied. However, normal (i.e., non-Mira-type) $M$ giants show variability amplitudes of $\Delta V=1-2 \mathrm{mag}$, much smaller than those of Mira stars, without clear periodic variations. They are classified as semi-regular

* Based on AMBER observations made with the Very Large Telescope Interferometer of the European Southern Observatory. Program ID: 081.D-0233(A) (AMBER Guaranteed Time Observation). or irregular variables. Among the M stars listed in the General Catalog of Variable Stars (GCVS) 4.2 (Samus et al. 2009) are 1987 stars classified as semi-regular or irregular giants (denoted as SRA, SRB, SRD, LB, and L in the catalog), in contrast to 1524 stars classified as Mira variables (see, however, Lebzelter et al. 1995, for the uncertainty in the classification in the GCVS). There are also many semi-regular or irregular variables whose spectral type is unknown in the catalog. Therefore, normal M giants with small variability amplitudes may outnumber Mira stars considerably.

Despite their small variability amplitudes and the absence of clear periodicities, normal $\mathrm{M}$ giants are experiencing mass loss with mass-loss rates comparable to those in (optically bright) Mira stars. Normal M giants in the AGB phase show massloss rates of $10^{-7}-10^{-6} M_{\odot} \mathrm{yr}^{-1}$ and expansion velocities of 5-15 $\mathrm{km} \mathrm{s}^{-1}$ (e.g., Gonzalez Delgado et al. 2003; Winters et al. 2003; De Beck et al. 2010). There is also evidence of significant mass loss in RGB stars with mass-loss rates up to $10^{-6} M_{\odot} \mathrm{yr}^{-1}$ 
A\&A 537, A53 (2012)

Table 1. Summary of the AMBER observations of BK Vir with the E0-G0-H0 AT configuration.

\begin{tabular}{cccccccccc}
\hline \hline$\#$ & Night & $\begin{array}{c}t_{\text {obs }} \\
(\mathrm{UTC})\end{array}$ & $\begin{array}{c}B_{\mathrm{p}} \\
(\mathrm{m})\end{array}$ & $\begin{array}{c}\text { PA } \\
\left({ }^{\circ}\right)\end{array}$ & $\begin{array}{c}\text { Seeing } \\
\left({ }^{\prime \prime}\right)\end{array}$ & Airmass & $\begin{array}{c}\text { DIT } \\
(\mathrm{s})\end{array}$ & $\begin{array}{c}\text { Number } \\
\text { of frames }\end{array}$ & Calibrator \\
\hline 1 & 2009 Jan. 19 & $07: 43: 32$ & $13.9 / 27.9 / 41.9$ & 73 & 0.9 & 1.2 & 6 & 75 & $v \mathrm{Hya}^{\dagger}$ \\
2 & 2009 Feb. 28 & $06: 15: 16$ & $15.6 / 31.2 / 46.8$ & 74 & 0.7 & 1.1 & 6 & 75 & $v \mathrm{Hya}^{\dagger}$ \\
3 & 2009 Apr. 15 & $03: 43: 30$ & $15.9 / 31.8 / 47.8$ & 74 & 0.8 & 1.1 & 6 & 75 & $\beta$ Crv \\
\hline
\end{tabular}

Notes. ${ }^{(\dagger)}$ Means that the calibrator was observed before and after the science target. Seeing is in the visible.

near the tip of the RGB (Origlia et al. 2007, 2010; Ita et al. 2007). The mass-loss rate $(\dot{M})$ and expansion velocity $\left(v_{\exp }\right)$ of our target, BK Vir, are estimated to be $(1.5-4) \times 10^{-7} M_{\odot} \mathrm{yr}^{-1}$ and $4-7.5 \mathrm{~km} \mathrm{~s}^{-1}$, respectively (Gonzalez Delgado et al. 2003; Winters et al. 2003), which are approximately the same as in the prototypical Mira $o$ Cet with $\dot{M}=(1-8) \times 10^{-7} M_{\odot} \mathrm{yr}^{-1}$ and $v_{\text {exp }}=1.5-7 \mathrm{~km} \mathrm{~s}^{-1}$ (Winters et al. 2003). This illustrates the importance of the contribution of normal $\mathrm{M}$ giants to the chemical enrichment of the interstellar medium.

For understanding the mass-loss mechanism in red giant stars, it is indispensable to probe the physical properties of the region between the upper photosphere and the innermost part of the circumstellar envelope, where the energy and momentum are expected to be deposited for the wind acceleration. It is now known from spectroscopy and high-spatial resolution observations in the IR that there is a dense, warm $(\sim 1000-2000 \mathrm{~K})$ molecular outer atmosphere, the so-called MOLsphere, extending to a few $R_{\star}$ not only in Mira stars but also in normal K-M giants with small variability amplitudes (e.g., Tsuji et al. 1997; Tsuji 2001; Perrin et al. 2004; Ohnaka 2004; Ohnaka et al. 2005; Takami et al. 2009). This MOLsphere is considered to play an important role in driving mass outflows. In the case of Mira stars, the large-amplitude pulsation can fairly explain the presence of the extended atmosphere (e.g., Ohnaka et al. 2006; Wittkowski et al. 2007, 2008; Woodruff et al. 2009). However, the amplitudes of the stellar pulsation are much smaller in normal K-M giants, and therefore, the origin of the MOLsphere in these stars is by no means clear. In addition to the MOLsphere, the detection of UV emission lines as well as the $\mathrm{H} \alpha$ line suggests the presence of a chromosphere (e.g., Eaton 1995; Isabel Pérez Martínez et al. 2011, and references therein). This means that there are both hot and warm components in the outer atmosphere of normal $\mathrm{K}-\mathrm{M}$ giants, and the spectral analyses of the CO lines in the UV and IR support this picture (e.g., Wiedemann et al. 1994; McMurry \& Jordan 2000).

The multi-component nature of the outer atmosphere is a key to understanding the mass-loss mechanism in red giants. Infrared spectro-interferometry, which enables spatially resolved spectroscopy of molecular or atomic spectral features with a spatial resolution much higher than achieved with singledish telescopes, is effective for probing the physical properties of the outer atmosphere. While Mira stars have been intensively studied by IR spectro-interferometry as mentioned above, IR spectro-interferometric observations of normal red giants are still very scarce. Recently, Martí-Vidal et al. (2011) have presented near-IR spectro-interferometric observations of the M6/7 giant RS Cap in the CO first overtone bands and $\mathrm{H}_{2} \mathrm{O}$ bands with a medium spectral resolution of 1500 using VLTI/AMBER. In this paper, we report on VLTI/AMBER observations of the M7 giant BK Vir in the individual $\mathrm{CO}$ first overtone lines near $2.3 \mu \mathrm{m}$ with a higher spectral resolution of 12000 . BK Vir shows no peculiar features and, therefore, is a good representative of normal $\mathrm{M}$ giants (its evolutionary status is discussed in Sect. 4.1). It was selected also for technical reasons, such as the brightness and angular size appropriate for AMBER observations.

The paper is organized as follows. Our AMBER observations and data reduction are described in Sect. 2. The observational results are shown in Sect. 3 followed by the modeling of the data presented in Sect. 4. The implications of the results of the modeling are discussed in Sect. 5. Conclusions are presented in Sect. 6.

\section{Observations}

AMBER (Petrov et al. 2007) is the near-IR (1.3-2.4 $\mu \mathrm{m}$ ) spectro-interferometric instrument at VLTI. It enables us to obtain information on the amplitude and phase of the complex Fourier transform of the object's intensity distribution with a spectral resolution of up to 12000 by combining three $8.2 \mathrm{~m}$ unit telescopes (UTs) or $1.8 \mathrm{~m}$ auxiliary telescopes (ATs). AMBER measures several quantities related to the complex Fourier transform of the object's intensity distribution: visibility, differential phase (DP), and closure phase (CP). The visibility (also called visibility amplitude) contains information about the size and shape of the object. The DP can measure the deviation of the object's phase in a spectral feature from that in the continuum, and non-zero DP within a spectral feature means that the spectral feature-forming region is asymmetric. The $\mathrm{CP}$ is the sum of the measured phases around a closed triangle of baselines. The CP is always zero or $\pi$ for point-symmetric objects, and non-zero and non- $\pi$ CPs indicate an asymmetry in the object.

BK Vir was observed at three epochs between January and April 2009 with AMBER using the E0-G0-H0 telescope configuration with ATs, as summarized in Table 1. We used the highspectral resolution mode in the $K$-band (HR_K) with a spectral resolution of 12000 in the wavelength range between 2.26 and $2.31 \mu \mathrm{m}$, which includes the $\mathrm{CO}(2,0)$ band head at $2.294 \mu \mathrm{m}$. The VLTI fringe-tracker FINITO was used to stabilize the fringes for a detector integration time (DIT) of $6 \mathrm{~s}$. This long DIT was necessary to achieve reasonable signal-to-noise ratios $(\mathrm{S} / \mathrm{N})$ in the $\mathrm{CO}$ lines, where the correlated flux (i.e., flux $\times$ visibility amplitude) is very low.

We reduced the data with amdlib ver $2.2^{1}$. We took the best $80 \%$ of the frames in terms of the fringe $\mathrm{S} / \mathrm{N}$ (Tatulli et al. 2007). The interferometric calibrators ( $v$ Hya and $\beta$ Crv) were observed just before and/or after BK Vir. We adopted angular diameters of 3.40 and 4.45 mas for $v$ Hya and $\beta$ Crv, respectively (Richichi $\&$ Perschron 2005). The errors of the calibrated visibilities, DPs, and CPs were estimated as described in Ohnaka et al. (2009).

The AMBER data taken in HR_K mode before December 2009 are affected by the Fabry-Perot effect, which is

\footnotetext{
1 Available at http://www.jmmc.fr/data_processing_amber. htm
} 
caused by the dichroics of the InfraRed Image Sensor (IRIS), which stabilizes the image motion ${ }^{2}$. This effect appears as timedependent, high-frequency beating in raw visibilities, DPs, and CPs, when plotted as a function of wavelength. Fortunately, this effect is mostly removed by using the calibrators observed just before and/or after the science target, although the residual of this effect appears as the small-amplitude beating in some cases (as seen in the observed CP at wavelengths below $2.293 \mu \mathrm{m}$ in Fig. 1e).

The long DIT of $6 \mathrm{~s}$ makes the absolute calibration of the observed visibilities uncertain, because we cannot know the fraction of time during DIT when the fringes were lost, and the fringe tracking may work differently for the science target and the calibrator. Therefore, we took the following approach for the absolute calibration of the observed visibilities. We note that the $K$-band uniform-disk diameters of BK Vir reported in the literature show little time variation within the measurement errors: $11.2 \pm 0.6$ mas (Dyck et al. 1998), $10.73 \pm 0.23$ mas (Perrin et al. 1998), and $11.49 \pm 0.40$ mas (Perrin et al. 2003). In the present work, we adopted a uniform-disk diameter of 10.73 mas, which has the smallest error among the above measurements. We scaled the observed calibrated visibilities in the continuum to those expected from the uniform disk with an angular diameter of 10.73 mas.

The wavelength calibration was carried out using the telluric lines identified in the spectra of the calibrators. As a template of the telluric lines, we convolved the atmospheric transmission spectrum measured at the Kitt Peak National Observatory ${ }^{3}$ to match the spectral resolution of AMBER. The uncertainty in wavelength calibration is $4 \times 10^{-5} \mu \mathrm{m}\left(\sim 5.2 \mathrm{~km} \mathrm{~s}^{-1}\right)$. The wavelength scale was converted to the laboratory frame using a heliocentric velocity of $16.5 \mathrm{~km} \mathrm{~s}^{-1}$ measured for BK Vir (Gontcharov 2006; Famaey et al. 2005).

Because no spectroscopic standard star was observed, we used the interferometric calibrators for the spectroscopic calibration to remove the telluric lines from the observed spectrum of BK Vir. However, both $\beta \mathrm{Crv}$ (G5II) and $v$ Hya (K0/K1III) show $\mathrm{CO}$ absorption lines. In this case, the calibrated spectrum of the science target is derived as $F_{\star}^{\mathrm{sci}}=F_{\mathrm{obs}}^{\mathrm{sci}} /\left(F_{\mathrm{obs}}^{\mathrm{cal}} / F_{\star}^{\mathrm{cal}}\right)$, where $F_{\star}^{\text {sci(cal) }}$ and $F_{\text {obs }}^{\text {sci(cal) }}$ denote the true and observed (i.e., including the atmospheric transmission and the detector's response) spectra of the science target (or calibrator), respectively. To estimate the true spectrum of $v$ Hya, we used the high-resolution $K$-band spectrum of $\alpha$ Boo (K1III) obtained by Wallace \& Hinkle (1996), which has a similar spectral type to $v$ Hya. The spectrum of $\alpha$ Boo, which has a spectral resolution of 89000 , was convolved down to match the resolution of AMBER. This convolved spectrum of $\alpha$ Boo reproduces the strength of the $\mathrm{CO}$ band head observed in $v$ Hya, indicating that it can be used as the true spectrum of $v$ Hya.

For $\beta$ Crv, however, no high-resolution $\mathrm{CO}$ spectra of stars with similar spectral types are available in the literature. Therefore, we estimated the true spectrum for $\beta \mathrm{Crv}$ by constructing a model $\mathrm{CO}$ spectrum using the MARCS models (Gustafsson et al. 2008). The MARCS models represent spherically symmetric photospheres with molecular and atomic line opacities taken into account using the opacity sampling method. Each MARCS model is specified by effective temperature $\left(T_{\text {eff }}\right)$, surface gravity $(g)$, microturbulent velocity $\left(v_{\text {micro }}\right)$,

\footnotetext{
2 AMBER data taken after the replacement of the IRIS dichroics in December 2009 do not show this effect any more.

${ }^{3}$ http://www. eso.org/sci/facilities/paranal/

instruments/isaac/tools/spectra/atmos_S_K.fits
}

chemical composition, and stellar mass $\left(M_{\star}\right)$. These parameters derived for $\beta \mathrm{Crv}$ are $T_{\mathrm{eff}}=5100-5145 \mathrm{~K}, \log g=2.2-2.7$ ( $g$ is given in units of $\mathrm{cm} \mathrm{s}^{-2}$ throughout the paper), $v_{\text {micro }}=$ 1.5-2.1 $\mathrm{km} \mathrm{s}^{-1}$, and 3.0-3.7 $M_{\odot}$ (Luck et al. 1995; Takeda et al. 2008; Lyubimkov et al. 2010). The CNO abundances derived by Luck et al. (1995) suggest the mixing of CN-cycled material. We selected a model atmosphere with parameters as close to these values as possible from the MARCS model grid $^{4}$. The selected model is characterized with $T_{\text {eff }}=5250 \mathrm{~K}, \log g=2.5$, $v_{\text {micro }}=2 \mathrm{~km} \mathrm{~s}^{-1}, M_{\star}=1 M_{\odot}$, and "moderately CN-cycled" chemical composition with $\mathrm{C} / \mathrm{N}=1.5$. The stellar mass of this model is smaller than the observationally estimated values above. However, the atmosphere is approximately plane-parallel with a geometrical thickness of $\sim 1 \%$ of the stellar radius. In this case, the stellar mass does not affect the atmospheric structure noticeably. Using the temperature and pressure distributions of this model, we computed a synthetic spectrum for the $\mathrm{CO}$ first overtone lines using the line list of Goorvitch (1994). The carbon abundance was adjusted to fit the $\mathrm{CO}$ band head in the uncalibrated spectrum of $\beta$ Crv. With $F_{\star}^{\text {cal }}$ constructed in this fashion, the calibrated spectrum of BK Vir was derived using the above equation. Although the strengths of the $\mathrm{CO}$ lines in two calibrators are very different (the line depths are $20-30 \%$ and $10 \%$ for $v$ Hya and $\beta$ Crv, respectively), the spectra of BK Vir calibrated with these stars agree well (see Fig. 3a, where both spectra calibrated with $v$ Hya (2009 Feb. 28) and $\beta$ Crv (2009 Apr. 15) are shown). This indicates that the calibrated CO line spectra of BK Vir are not significantly affected by the above, unusual spectroscopic calibration.

\section{Results}

Figure 1 shows the observed visibilities, DPs, CPs, and spectrum from data set \#3. The results from the other data sets are similar, and are therefore not shown. The figure reveals significant signatures of the CO lines in the observed visibilities, DPs, and CPs. Thanks to the long DIT of $6 \mathrm{~s}$, visibilities as low as 0.01 could be measured in the $\mathrm{CO}$ lines. As plotted in Fig. 1d, the uniformdisk diameter obtained from the observed visibilities shows that the star appears larger in the CO lines (12-14 mas) than in the continuum (10.73 mas) by $12-31 \%$. However, the uniform-disk fit in the CO lines is poor with reduced $\chi^{2}$ values much higher than 1 (as high as $10^{2}-10^{3}$ ), which means that the uniform disk is a poor representation of the object's shape in the CO lines. Therefore, the uniform-disk diameter in the $\mathrm{CO}$ lines should be regarded as a very rough estimate of the characteristic size of the object.

Figures 1e-h show the detection of non-zero DPs (particularly clear on the $47.8 \mathrm{~m}$ baseline) and non-zero/non- $\pi$ CPs in the $\mathrm{CO}$ lines, which indicate an asymmetry in the CO lineforming region. High-spatial resolution observations have revealed that asymmetries in the atmosphere of red giant stars are common. The images of the Mira star R Cas in the TiO bands obtained by Weigelt et al. (1996) show elongation, while the image in the continuum appears to be circular. The optical images of the prototypical Mira star $o$ Cet taken with the Hubble Space Telescope show significant asymmetries (Karovska et al. 1997). Tuthill et al. (1999) also revealed departures from circular symmetry in five Mira stars (including the above two stars) in the optical and near-IR. The aperture synthesis images of the Mira stars R Aqr and U Ori in the $H$-band obtained with the Infrared Optical Telescope Array (IOTA) show asymmetric,

\footnotetext{
4 http://marcs.astro.uu.se
} 

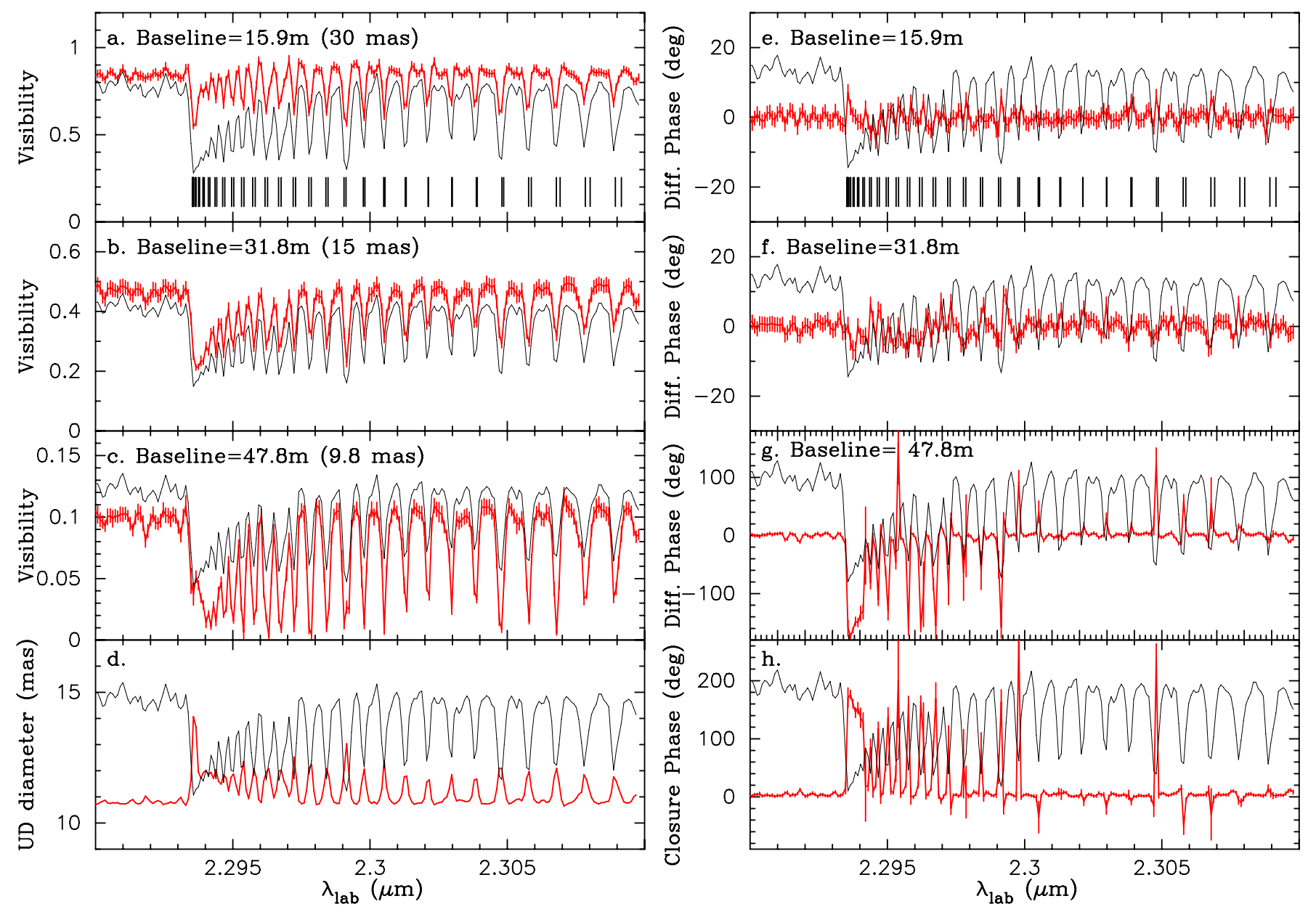

Fig. 1. AMBER data of BK Vir (data set \#3). In each panel, the scaled observed spectrum is plotted by the black solid lines. a)-c) Visibilities observed on the 15.9, 31.8, and $47.8 \mathrm{~m}$ baselines (red lines). The corresponding spatial resolutions are also given. d) Uniform-disk diameter (red line) derived by fitting the visibilities shown in panels a)-c). e)-g) Differential phases observed on the 15.9, 31.8, and 47.8 $\mathrm{m}$ baselines (red lines). h) Closure phase (red line).

extended atmospheres (Ragland et al. 2008; Pluzhnik et al. 2009). U Ori shows asymmetry in the $K$-band as well (Mondal \& Chandrasekhar 2004). There are more stars for which asymmetries are indicated from the detection of non-zero CPs. Bedding et al. (1997) detected non-zero CPs for the Mira-like star R Dor. Ragland et al. (2006) also report asymmetries in a sample of AGB stars based on the detection of non-zero CPs. More recently, Wittkowski et al. (2011) have shown asymmetries in the CO first overtone bands in Mira stars with AMBER observations with a spectral resolution of 1500. Our AMBER observations are the first study to detect asymmetry in a red giant star in the individual $\mathrm{CO}$ lines.

Figure $2 \mathrm{a}$, which shows an enlarged view of the visibilities in four representative $\mathrm{CO}$ lines obtained on the $47.8 \mathrm{~m}$ baseline, reveals that the observed visibilities are approximately symmetric with respect to the line center. On the other hand, Fig. $2 b$ shows the visibility of the red supergiant Betelgeuse observed on the $11.54 \mathrm{~m}$ baseline (data set \#5 of Ohnaka et al. 2011). Because the angular diameter of Betelgeuse (42.5 mas, Ohnaka et al. 2011) is four times larger than that of BK Vir (10.73 mas), the $11.54 \mathrm{~m}$ baseline for Betelgeuse probes nearly the same spatial scale in terms of the stellar angular diameter as the $47.8 \mathrm{~m}$ baseline for BK Vir $(0.9 \times$ stellar angular diameter). Figure $2 \mathrm{~b}$ clearly shows that the observed visibilities are asymmetric with respect to the line center in the case of
Betelgeuse: the visibilities are characterized by the minima in the blue wing and the maxima in the red wing. These asymmetric visibilities are interpreted as temporally variable, inhomogeneous gas motions within 1.3-1.4 $R_{\star}$ over the stellar surface with velocity amplitudes of up to $20-30 \mathrm{~km} \mathrm{~s}^{-1}$ (Ohnaka et al. 2009, 2011). The absence of such asymmetric visibilities in BK Vir suggests that the amplitude of the inhomogeneous velocity field is much smaller than AMBER's spectral resolution of 12000 , say, $\sim 5 \mathrm{~km} \mathrm{~s}^{-1}$, and/or the spatial scale of the inhomogeneous gas motions is much smaller than the spatial resolution of the current AMBER data (9.8 mas). However, the former possibility is more favorable, because the non-zero DPs and nonzero/non- $\pi$ CPs shown in Fig. 1 indicate that the inhomogeneity is spatially resolved with the current spatial resolution.

We examined time variation using the AMBER data taken at nearly the same $u v$ points. The shortest and middle baselines of the data sets taken on 2009 Feb. 28 and 2009 Apr. 15 are almost the same (the $1 \mathrm{~m}$ difference in the longest baseline is too significant to study time variations). A comparison between these two data sets is shown in Fig. 3. The sampled wavelengths are slightly different for the two nights because of the difference in the correction to convert the observed wavelength scale to the heliocentric scale. Figures $3 \mathrm{a}$ and $\mathrm{c}$ show that the observed spectra and the visibilities on the $32 \mathrm{~m}$ baseline measured on the two nights form continuous curves, suggesting no time variation. 


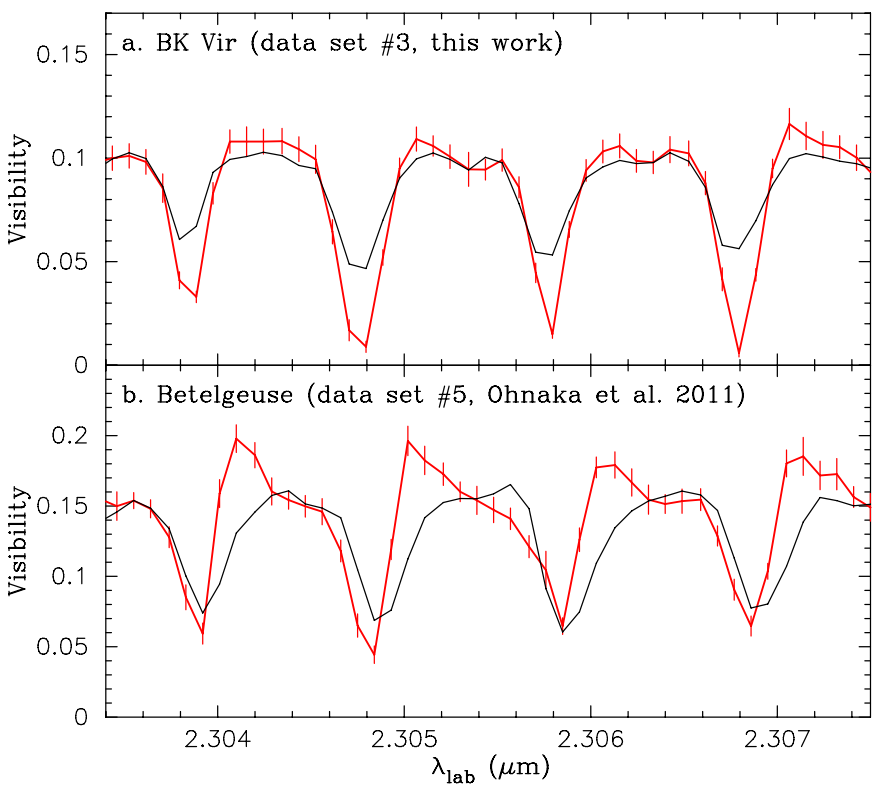

Fig. 2. Visibilities in four representative CO first overtone lines in the red giant BK Vir (panel a)) and the red supergiant Betelgeuse (panel b)). In both panels the black solid lines represent the scaled observed spectra. The red solid lines represent the visibility obtained for BK Vir on the $47.8 \mathrm{~m}$ baseline (panel a)) and that obtained for Betelgeuse on the $11.54 \mathrm{~m}$ baseline (panel b)).

This is also the case for the $16 \mathrm{~m}$ visibility shown in Fig. 3b except for the CO band head at $2.2936 \mu \mathrm{m}$, where the visibility measured on Apr. 15 is $12-20 \%$ lower than that measured on Feb. 28. This suggests possible time variation on a spatial scale of 30 mas $(3 \times$ stellar diameter $)$. The decrease in the visibility corresponds to an increase in the uniform-disk diameter by $22 \%$ (from 16 to 19.5 mas) or an increase in the Gaussian FWHM by $14 \%$ (from 10.5 to 12 mas) between Feb. 28 and Apr. 15. However, the difference is within $3-\sigma$ of the data from Feb. 28. Therefore, more observations are needed to definitively confirm time variations in the visibility.

\section{Modeling of the AMBER data}

\subsection{Determination of stellar parameters}

To interpret the AMBER observations, we used MARCS models. As mentioned in Sect. 2, these models assume spherical symmetry. While the asymmetry in the CO-line-forming region is detected, we used these spherical models as the first approximation. To select a MARCS model appropriate for BK Vir, we determined its stellar parameters $\left(T_{\text {eff }}, g, v_{\text {micro }}, M_{\star}\right.$, and chemical composition) as follows.

We estimated the effective temperature from the observed angular diameter and the bolometric flux obtained by integrating the photometric data available in the literature (Two-Micron Sky Survey, Neugebauer \& Leighton 1969; NOMAD Catalog, Zacharias et al. 2004; 2MASS, Skrutskie et al. 2006; Fouque et al. 1992; Kerschbaum \& Hron 1994; IRAS Point Source Catalog). The photometric data were corrected for the interstellar extinction using $E(B-V)=0.023$ (Schlegel et al. 1998) and assuming $A_{V}=3.1 E(B-V)$. Combining the angular diameter of 10.73 mas adopted in Sect. 2 and a derived bolometric flux of $2.80 \times 10^{-9} \mathrm{Wm}^{-2}$ results in an effective temperature of $2920 \mathrm{~K}$. While this agrees with the effective temperature of $3074 \pm 141 \mathrm{~K}$ derived by Dyck et al. (1998), they obtained a higher bolometric
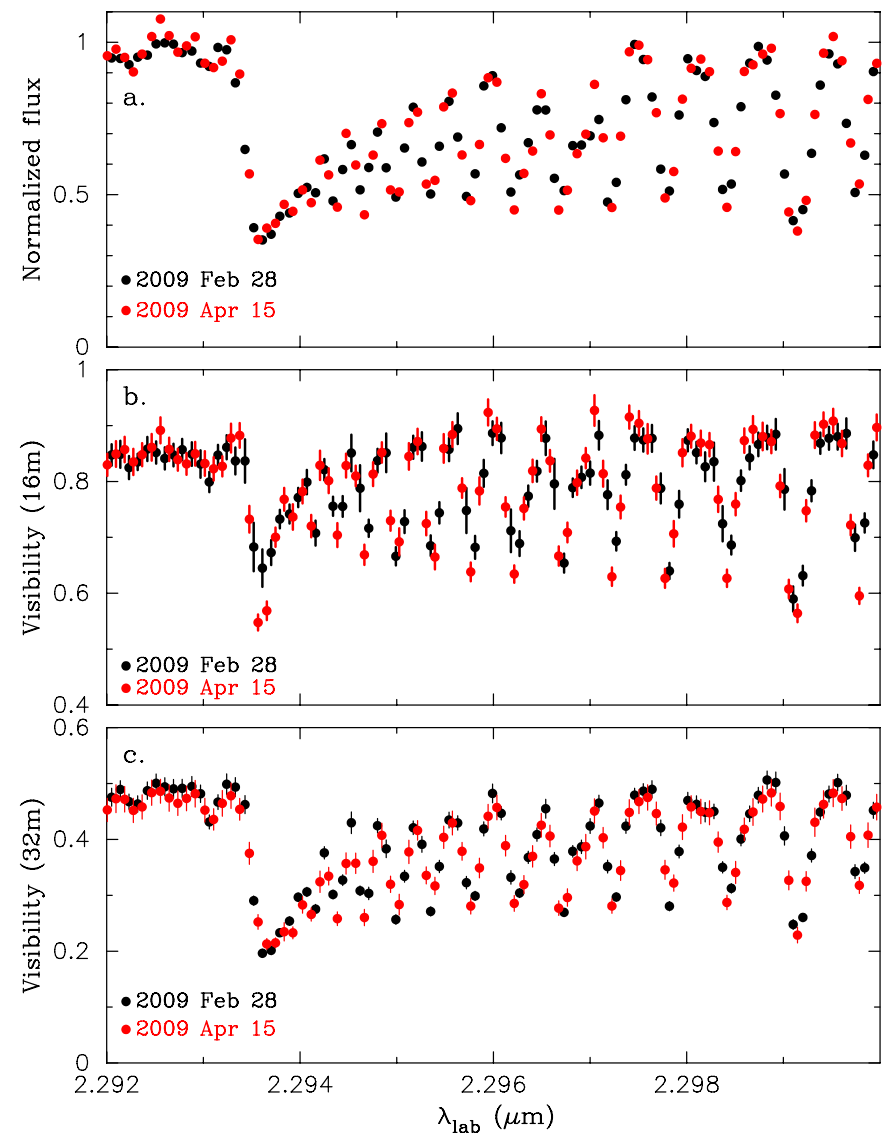

Fig. 3. Spectra (panel a)) and visibilities (panels b): $16 \mathrm{~m}$ baseline and c): $32 \mathrm{~m}$ baseline) obtained on 2009 Feb. 28 and 2009 Apr. 15. In all panels, the data taken on 2009 Feb. 28 and 2009 Apr. 15 are plotted by the black and red dots, respectively.

flux of $3.9 \times 10^{-9} \mathrm{Wm}^{-2}$. If we adopt as the uncertainty in the bolometric flux half of the difference between the values from Dyck et al. (1998) and from the present work, the total error (i.e., error resulting from the uncertainties in the angular diameter and in the bolometric flux) in our effective temperature is $\pm 150 \mathrm{~K}$. Using the distance of $180 \mathrm{pc}$ based on the Hipparcos parallax of $5.53 \pm 0.68$ mas (van Leeuwen 2007) and the above bolometric flux of $2.80 \times 10^{-9} \mathrm{Wm}^{-2}$, we derived a luminosity of $2700 L_{\odot}\left(M_{\mathrm{BOL}}=-3.8\right)$. With the error in the parallax and the above error in the bolometric flux, the total uncertainty in the luminosity is $\pm 850 L_{\odot}$.

To estimate the stellar mass, we compared the position of BK Vir on the H-R diagram with theoretical evolutionary tracks. Figure 4 shows evolutionary tracks for 1 and $2 M_{\odot}$ stars (Bertelli et al. 2008; and Herwig 2005 ${ }^{5}$, respectively), together with the observationally derived position of BK Vir. The figure suggests that the mass of BK Vir is close to $1 M_{\odot}$. The adoption of a stellar mass of $1 M_{\odot}$ translates into a surface gravity of $\log g=-0.17$ with a stellar radius of $201 R_{\odot}(0.94 \mathrm{AU})$.

Whereas BK Vir is classified as an AGB star in most of the literature, Fig. 4 shows that the luminosity of BK Vir is just at the tip of the RGB. Moreover, Lebzelter \& Hron (1999) report negative detection of ${ }^{99} \mathrm{Tc}$, which would be strong evidence of the third dredge-up in the AGB. Therefore, we cannot conclude whether BK Vir is an RGB star or an early-AGB star that has not yet experienced the third dredge-up. In either case, however, the surface chemical composition is expected to be changed by the

5 http://astrowww.phys.uvic.ca/ fherwig 


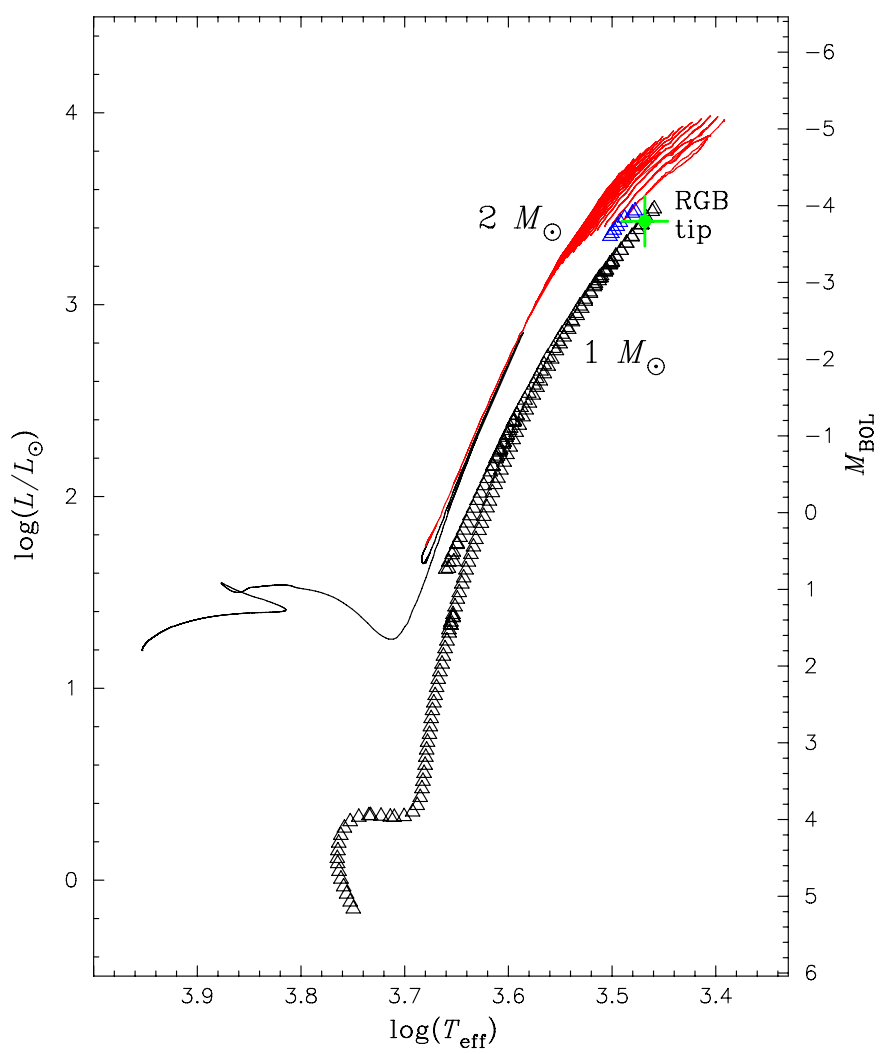

Fig. 4. Observationally derived position of BK Vir on the H-R diagram (green dot with the error bars). The open triangles and solid line represent the evolutionary tracks for a 1 and $2 M_{\odot}$ star from Bertelli et al. (2008) and Herwig (2005), respectively. The AGB stage (early AGB and thermally-pulsing AGB) is marked in blue and red for the evolutionary tracks for $1 M_{\odot}$ and $2 M_{\odot}$, respectively.

first dredge-up in the RGB phase, which mixes the CN-cycled material to the stellar surface. This makes the MARCS models with the "moderately CN-cycled" chemical composition (with $[\mathrm{Fe} / \mathrm{H}]=0.0)$ suitable for BK Vir.

No analysis of the micro-turbulent velocity in BK Vir is available in the literature. However, the high-resolution spectroscopic analysis of M7-8 giants with $T_{\text {eff }} \lesssim 3000 \mathrm{~K}$ by Tsuji (2008) shows micro-turbulent velocities of $3-4 \mathrm{~km} \mathrm{~s}^{-1}$. We adopt these values for BK Vir as well.

\subsection{Comparison with the MARCS photospheric model}

The MARCS model with the parameters as close to the derived values as possible is characterized by $T_{\text {eff }}=3000 \mathrm{~K}$, $\log g=0.0, M_{\star}=1 M_{\odot}, v_{\text {micro }}=2 \mathrm{~km} \mathrm{~s}^{-1}$ (models with $v_{\text {micro }}=$ 3-4 $\mathrm{km} \mathrm{s}^{-1}$ are not available for this combination of $T_{\text {eff }}, \log g$, $M_{\star}$, and chemical composition, and the model with $v_{\text {micro }}=$ $2 \mathrm{~km} \mathrm{~s}^{-1}$ is the closest one). Using the temperature and pressure distributions of the downloaded MARCS model, we computed the monochromatic intensity profile and spectrum over the observed wavelength range using the CO line list of Goorvitch (1994), as described in Ohnaka et al. (2006). The monochromatic visibility was calculated by the Fourier transform of the monochromatic intensity profile. The intensity profile, visibility, and spectrum were then convolved with the AMBER's spectral resolution. The angular scale of the model was determined so that the uniform-disk diameter derived from the model visibilities in the continuum matches the adopted value of 10.73 mas.

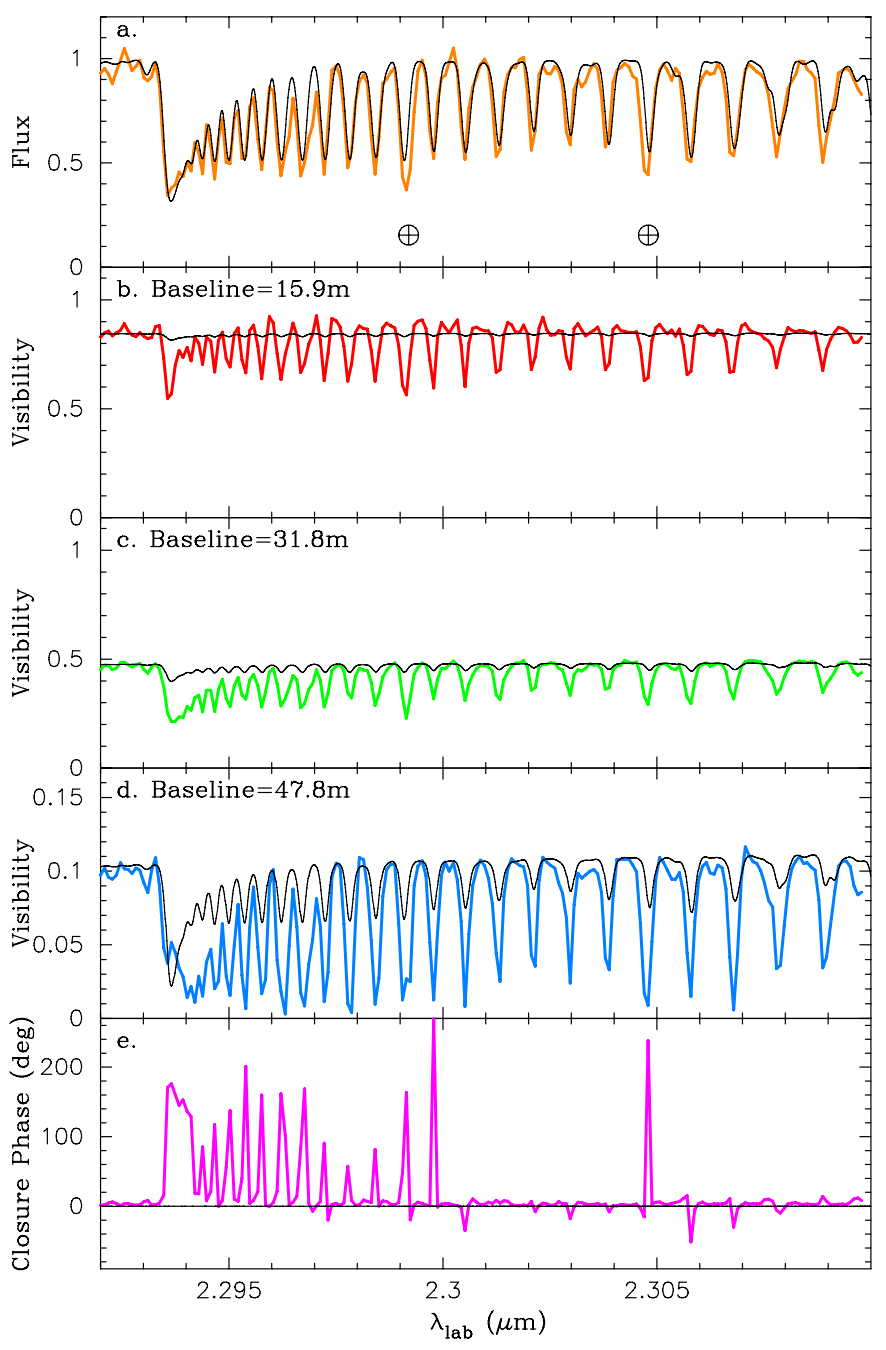

Fig. 5. Comparison between the observed interferometric data (data set \#3) and the MARCS model. In all panels, the black thin lines represent the predictions of the MARCS model with the parameters most appropriate for BK Vir (see Sect. 4.2). The thick colored lines represent the observed data. a) Spectrum. Residuals of the strong telluric lines are marked by $\oplus$. b) -d) Visibilities on the 15.9, 31.8, and $47.8 \mathrm{~m}$ baselines, respectively. e) Closure phase.

Figure 5 shows a comparison between the observed data (data set \#3) and the predictions by the MARCS photospheric model. While the observed CO spectrum is satisfactorily reproduced, the predicted visibilities in the $\mathrm{CO}$ lines are too high compared to the observed data. We also used other available MARCS models with slightly different stellar parameters $\left(T_{\mathrm{eff}}=\right.$ $2700-3100 \mathrm{~K}, \log g=-0.5-+0.5, M_{\star}=1-2 M_{\odot}$, and $v_{\text {micro }}=$ 2-5 $\mathrm{km} \mathrm{s}^{-1}$ ), but they cannot reproduce the observed visibilities in the $\mathrm{CO}$ lines, either. Figure 6 shows the intensity profiles and visibilities at three representative wavelengths in the continuum, at the $\mathrm{CO}$ band head, and in an isolated line. The figure illustrates that the photosphere extends to $1.17 R_{\star}$ in the CO band head and in the isolated line, but this geometrical extension is insufficient to explain the observed visibilities (Fig. 6c). The too high visibilities in the $\mathrm{CO}$ lines predicted by the MARCS model means the actual CO-line-forming layer is much more extended than the model predicts or there is an additional component contributing to the $\mathrm{CO}$ lines above the photosphere modeled by MARCS. Therefore, our AMBER observations have spatially resolved the MOLsphere in a normal $\mathrm{M}$ giant for the first time 

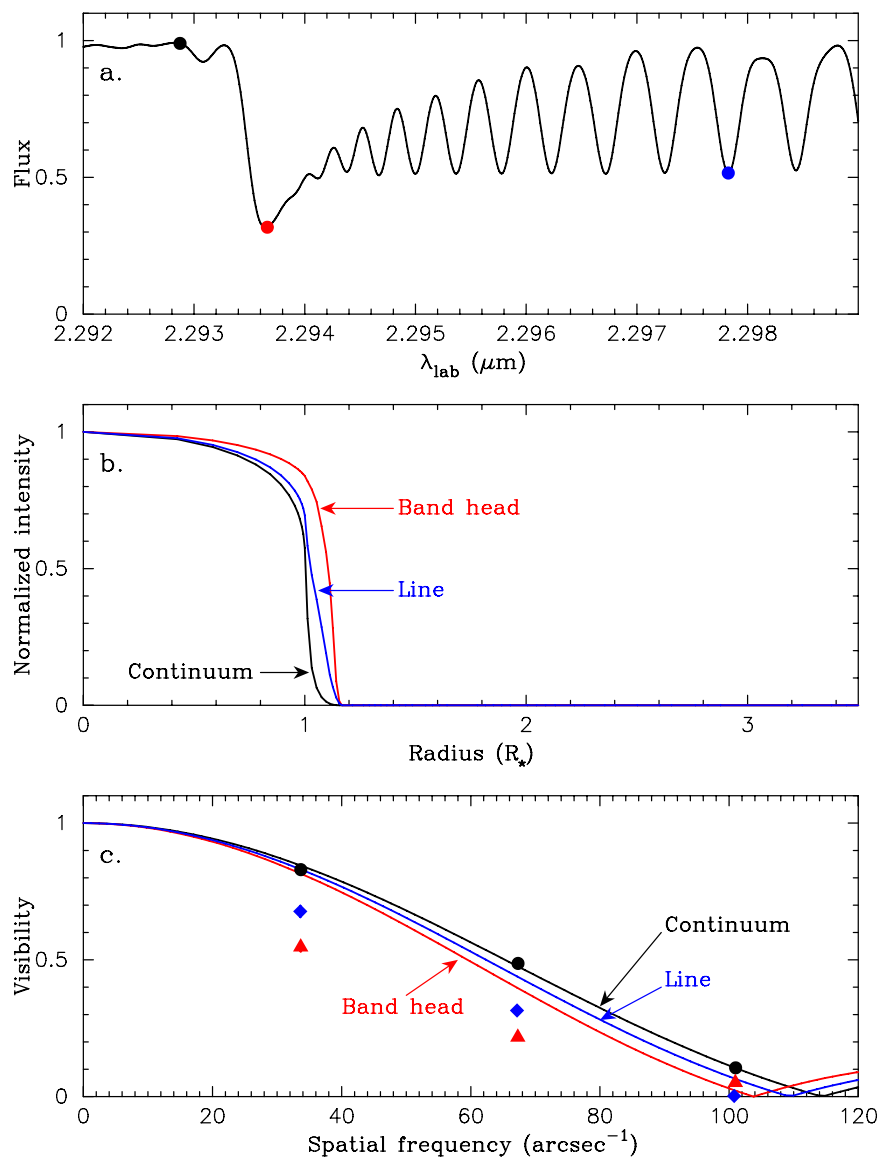

Fig. 6. MARCS model for BK Vir. a) Model spectrum near the CO band head. The black, red, and blue dots represent the wavelengths of the intensity profiles and visibilities shown in panels b) and c). b) Model intensity profiles for the continuum (black), CO band head (red) and an isolated CO line (blue). c) Model visibilities for the continuum, $\mathrm{CO}$ band head, and an isolated CO line are shown by the black, red, and blue solid lines, respectively. The filled dots, triangles, and diamonds represent the observed visibilities in the continuum, $\mathrm{CO}$ band head, and the isolated CO line (data set \#3).

in the individual CO lines. The same trend was reported for the normal M6-7 giant RS Cap by Martí-Vidal et al. (2011) based on AMBER observations of the $\mathrm{CO}$ bands with a lower spectral resolution of 1500. These studies illustrate that testing photospheric models only with the spectra of strong molecular or atomic features can be misleading.

\subsection{Modeling of the MOLsphere}

To characterize the physical properties of the MOLsphere, we interpreted the AMBER data with a model in which one or two extended $\mathrm{CO}$ layers with constant temperatures and densities are added above the MARCS photospheric model. This model is the same as that described in Ohnaka (2004), but with the following modification: each layer is assumed to be geometrically thin with a thickness of $0.1 R_{\star}$. The inner radius of each layer is used as the parameter to represent the size of the layer. The radius, temperature, and column density of each layer are changed as free parameters to fit the observed spectrum and visibilities simultaneously. We tentatively adopted a microturbulent velocity of $4 \mathrm{~km} \mathrm{~s}^{-1}$ for the $\mathrm{CO}$ layers based on the spectroscopic analysis of Tsuji (2008) for M7-8 giants. We first attempted to explain the observed data with models with one additional CO layer but could not reproduce the spectrum and visibilities simultaneously. Therefore, we attempted to explain the data with the models with two CO layers. Table 2 gives the range of the parameters searched in our modeling. Note that the radius of the inner layer was set to be equal to, or larger than, $1.2 R_{\star}$ to avoid an overlap with the MARCS photospheric model, which extends to $1.17 R_{\star}$.

Figure 7 shows a comparison between the observed data (data set \#3) and the best-fit model. The model intensity profiles and visibilities at three representative wavelengths (continuum, CO band head, and isolated CO line) are plotted in Fig. 8. This model is characterized by the inner CO layer at $1.2 R_{\star}$ with $2000 \mathrm{~K}$ and a CO column density of $2 \times 10^{22} \mathrm{~cm}^{-2}$ and the outer CO layer at $2.5 R_{\star}$ with $1900 \mathrm{~K}$ and a column density of $2 \times 10^{19} \mathrm{~cm}^{-2}$. The column densities of the inner and outer CO layer correspond to $16 \%$ and $0.016 \%$ of the CO column density in the MARCS photospheric model. As given in Table 2, we found the range of the radius, temperature, and $\mathrm{CO}$ column density of the inner layer to be $1.2-1.25 R_{\star}, 1900-2100 \mathrm{~K}$, and $(1-2) \times 10^{22} \mathrm{~cm}^{-2}$, respectively. The derived ranges for the radius, temperature, and $\mathrm{CO}$ column density of the outer layer are $2.5-3.0 R_{\star}, 1500-2100 \mathrm{~K}$, and $10^{19}-10^{20} \mathrm{~cm}^{-2}$, respectively. As shown in Fig. 7, the observed visibilities and spectrum are well reproduced. The model also predicts the $\mathrm{CP}$ to jump in the $\mathrm{CO}$ lines, in particular at the $\mathrm{CO}$ band head, as seen in the AMBER data. This can be explained as follows. Figure $8 \mathrm{~b}$ shows that the inner CO layer, which is optically thick, makes the star appear larger in the $\mathrm{CO}$ band head than in the continuum by $30 \%$. Then the longest baseline samples the second visibility lobe in the $\mathrm{CO}$ band head, as shown in Fig. 8c (red line and red triangle at a spatial frequency of $\sim 100 \operatorname{arcsec}^{-1}$ ). The phase in the second visibility lobe is $\pi$, while the phases on the shortest and middle baselines in the first visibility lobe are zero. This results in a predicted $\mathrm{CP}$ of $\pi$ for the $\mathrm{CO}$ band head and leads to the jumps in the $\mathrm{CP}$ as observed. Because the model is spherically symmetric, the observed non-zero/non- $\pi$ CPs and non-zero DPs are not reproduced ${ }^{6}$. However, the overall agreement in the spectrum, visibilities, and $\mathrm{CP}$ suggests that our model represents the approximate picture of the outer atmosphere of BK Vir.

It is impossible to uniquely constrain the detected inhomogeneous structure from our observations. However, we estimated the flux contribution of asymmetric features from the observed CPs as follows. We added a Gaussian-shaped spot to the bestfit model image in the $\mathrm{CO}$ lines and computed the visibility and $\mathrm{CP}$ at the observed baselines. We changed the size and intensity of the Gaussian-shaped spot so that the observed non-zero/non$\pi$ CPs $\left(40-100^{\circ}\right)$ can be reproduced without affecting the fit to the observed visibilities significantly. This experiment suggests that the flux contribution of the asymmetric feature is $3-5 \%$ of the total flux. These values agree with those estimated for other AGB stars. Ragland et al. (2006) derived a flux contribution of $3 \%$ from a geometrical modeling (uniform disk + unresolved spot) of the asymmetric features detected in a sample of AGB stars in the $H$-band. Wittkowski et al. (2011) also suggest a flux contribution of up to $\sim 3 \%$ in the $2 \mu \mathrm{m} \mathrm{H}_{2} \mathrm{O}$ band for the Mira star R Cnc.

The observed spectrum is nearly equally well reproduced by the MARCS-only model and MARCS+MOLsphere model, despite the clear disagreement between the observed visibilities and MARCS-only model. The reason is that the additional

${ }^{6}$ The model DPs for the shortest and middle baselines are zero, and the model DP for the longest baseline is the same as the model CP with its sign flipped. 
Table 2. Parameters of the MARCS+MOLsphere model for BK Vir.

\begin{tabular}{lcc}
\hline \hline Parameter & Searched range & Solution \\
\hline CO column density of the inner layer: $N_{\text {inner }}\left(\mathrm{cm}^{-2}\right)$ & $10^{21}, 2 \times 10^{21}, 5 \times 10^{21}, 10^{22}, \ldots, 10^{23}$ & $(1-2) \times 10^{22}$ \\
CO column density of the outer layer: $N_{\text {outer }}\left(\mathrm{cm}^{-2}\right)$ & $5 \times 10^{18}, 10^{19}, 2 \times 10^{19}, 5 \times 10^{19}, \ldots, 5 \times 10^{20}$ & $10^{19}-10^{20}$ \\
Temperature of the inner layer: $T_{\text {inner }}(\mathrm{K})$ & $1800 \ldots 2300\left(\Delta T_{\text {inner }}=100 \mathrm{~K}\right)$ & $1900-2100$ \\
Temperature of the outer layer: $T_{\text {outer }}(\mathrm{K})$ & $1200 \ldots 2300\left(\Delta T_{\text {outer }}=100 \mathrm{~K}\right)$ & $1500-2100$ \\
Radius the inner layer: $R_{\text {inner }}\left(R_{\star}\right)$ & $1.2,1.25,1.3$ & $1.2-1.25$ \\
Radius the outer layer: $R_{\text {outer }}\left(R_{\star}\right)$ & $1.5, \ldots, 3.5\left(\Delta R_{\text {outer }}=0.5 R_{\star}\right)$ & $2.5-3.0$ \\
\hline
\end{tabular}

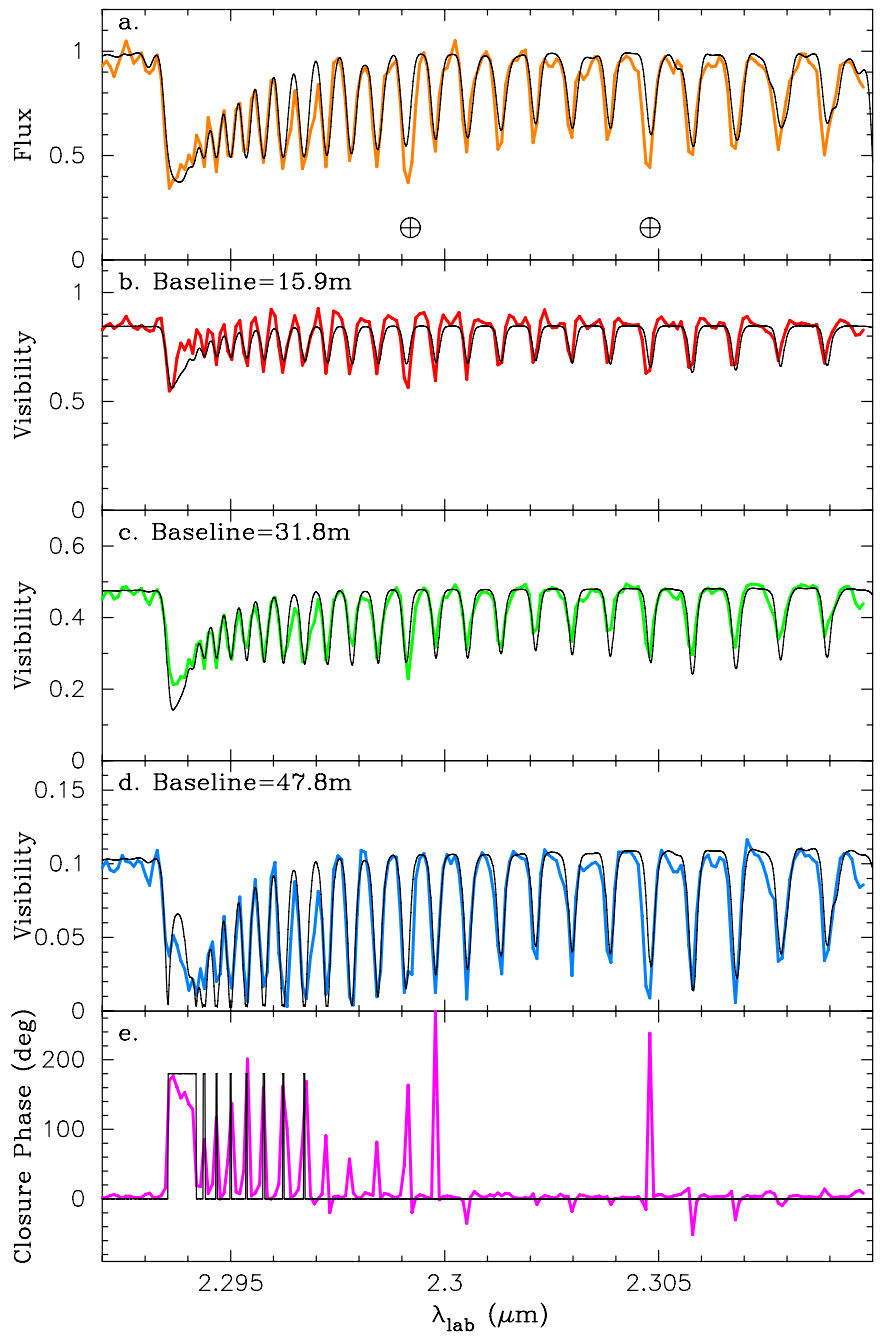

Fig. 7. Comparison between the observed interferometric data and the MARCS+MOLsphere model shown in the same manner as Fig. 5.

absorption due to the CO layers above the MARCS model is filled in by the extended emission of the same CO layers in the spatially unresolved spectrum. Therefore, good agreement between observation and model for spatially unresolved spectra of strong molecular features can be misleading, because these features can include the contribution from the extended outer atmosphere.

\section{Discussion}

Figure 9 shows the temperature distribution of the MARCS photospheric model as a function of radius, together with the location and the temperature of the extended $\mathrm{CO}$ layers derived from our modeling. The temperature of the inner $\mathrm{CO}$ layer
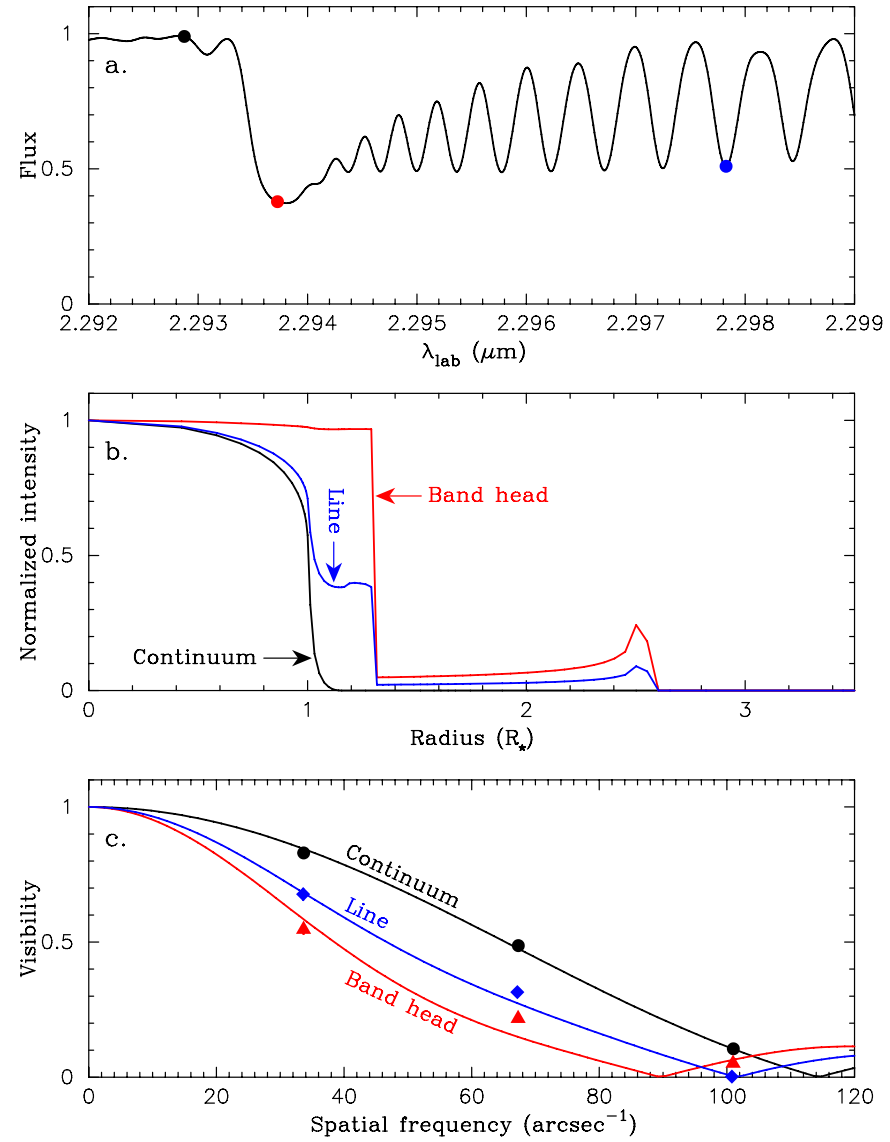

Fig. 8. MARCS+MOLsphere model for BK Vir shown in the same manner as Fig. 6.

is noticeably higher than that of the uppermost layer of the MARCS photospheric model, although the height is approximately the same. The temperature of the outer CO layer is also higher than, or equal to, that of the top of the photosphere. This suggests a temperature inversion in the MOLsphere of BK Vir.

Given that our spectroscopic calibration method without a usual spectroscopic standard star gives the reliable CO line spectra of BK Vir as described in Sect. 2, the uncertainty in the spectra are unlikely to affect the result of our modeling.

We checked whether this is merely a result of approximating the temperature distribution in the outer atmosphere by two discrete layers. We set the temperatures of the CO layers to be below $1500 \mathrm{~K}$, which is the temperature of the uppermost layer of the MARCS photospheric model, and attempted to reproduce the observed data. However, it turned out that these models cannot provide a reasonable fit to the observed spectrum and visibilities simultaneously. Therefore, the temperature inversion suggested in Fig. 9 is unlikely to be a result of the simplification we adopted for the structure of the outer atmosphere. 
K. Ohnaka et al.: Spatially resolving the M giant BK Vir in the CO first overtone lines

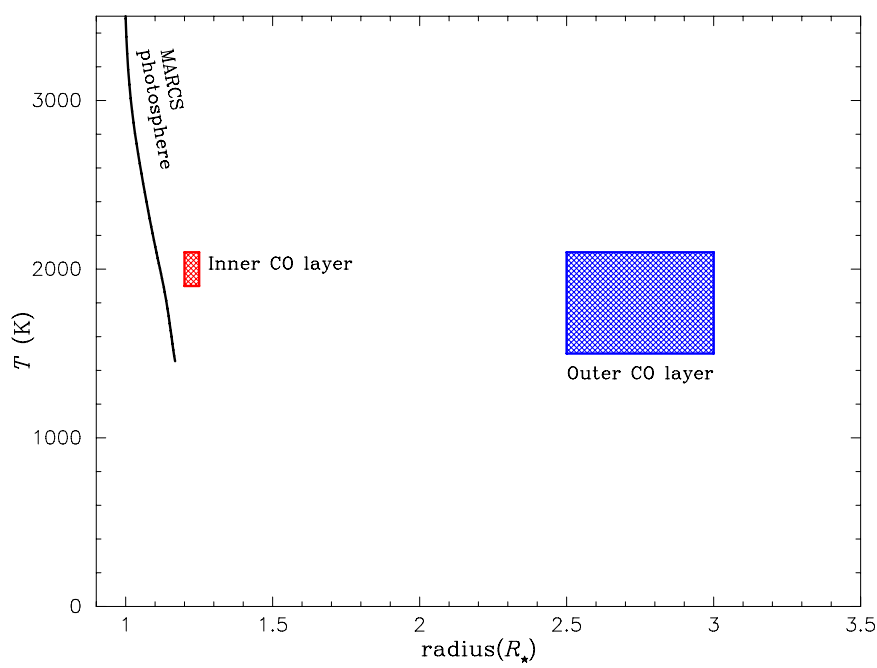

Fig. 9. Temperature distribution as a function of radius. The black solid line represents the temperature profile of the MARCS model used for BK Vir. The red and blue rectangles represent the ranges of the inner and outer CO layers derived from our modeling, respectively.

We also checked whether or not the assumption of local thermodynamical equilibrium (LTE) used in our model is valid for the CO layers. As described in Ohnaka (2004), we compared the collisional and radiative de-excitation rates. The collisional de-excitation rate $C_{\mathrm{ul}}$ is estimated as $N \sigma_{\mathrm{ul}} v_{\text {rel }}$, where $N$ is the number density of the primary collision partner, $\sigma_{\mathrm{ul}}$ is the cross section, which is approximated with the geometrical cross section, and $v_{\text {rel }}$ is the relative velocity between the collision partner and $\mathrm{CO}$ molecules. The $\mathrm{CO}$ column density of $10^{21} \mathrm{~cm}^{-2} \mathrm{de}-$ rived for the inner layer and the layer's geometrical thickness of $0.1 R_{\star}$ translates into a CO number density of $7 \times 10^{9} \mathrm{~cm}^{-3}$. In chemical equilibrium at the temperature of $2000 \mathrm{~K}$ derived for the inner layer, number densities of $\mathrm{H}$ atoms and $\mathrm{H}_{2}$ molecules of $1.2 \times 10^{13}$ and $1.4 \times 10^{13} \mathrm{~cm}^{-3}$ are necessary to obtain the above $\mathrm{CO}$ number density. We take both $\mathrm{H}$ atoms and $\mathrm{H}_{2}$ molecules as the primary collision partner of $\mathrm{CO}$ molecules, which leads to $N=2.6 \times 10^{13} \mathrm{~cm}^{-3}$. With a geometrical cross section $\sigma_{\mathrm{ul}}$ of $10^{-15} \mathrm{~cm}^{-2}$ and a relative velocity $v_{\text {rel }}$ of $5 \mathrm{~km} \mathrm{~s}^{-1}$ assumed, we obtain $C_{\mathrm{ul}}=1.3 \times 10^{4} \mathrm{~s}^{-1}$ for the inner CO layer. In the case of the outer $\mathrm{CO}$ layer, the lower limit of the $\mathrm{CO}$ column density $\left(10^{19} \mathrm{~cm}^{-2}\right)$ translates into a $\mathrm{CO}$ number density of $7.1 \times 10^{6} \mathrm{~cm}^{-3}$. For the temperatures of $1500-2100 \mathrm{~K} \mathrm{de}-$ rived for the outer layer, the number densities of $\mathrm{H}$ atoms and $\mathrm{H}_{2}$ molecules are $5.0 \times 10^{9}$ and $1.6 \times 10^{10} \mathrm{~cm}^{-3}(1500 \mathrm{~K})$ and $3.8 \times 10^{10}$ and $4.2 \times 10^{7} \mathrm{~cm}^{-3}(2100 \mathrm{~K})$, respectively. This leads to $N\left(\mathrm{H}+\mathrm{H}_{2}\right)=(2-4) \times 10^{10} \mathrm{~cm}^{-3}$. With the above $\sigma_{\text {ul }}$ and $v_{\text {rel }}$, we obtain $C_{\mathrm{ul}}=10 \mathrm{~s}^{-1}$ for the outer CO layer. These collisional de-excitation rates are much higher than radiative de-excitation rates $A_{\mathrm{ul}}$ of $0.6-1 \mathrm{~s}^{-1}$ found for the $\mathrm{CO}$ lines analyzed in the present work, which validates LTE for our modeling. Therefore, it is unlikely that the high temperatures derived for the $\mathrm{CO}$ layers are just a result of non-LTE effects.

The temperature inversion implies the operation of some heating mechanism in the outer atmosphere within $\sim 3 R_{\star}$. While the outwardly propagating shocks generated by the stellar pulsation can produce temperature jumps in Mira stars (e.g., Richter et al. 2003), it is not clear whether this is the case for normal $\mathrm{M}$ giants, where the variability amplitudes are much smaller. Despite the remarkable difference in the variability amplitude, BK Vir shows the temperature inversion in the molecular component of the outer atmosphere. This implies that the stellar pulsation may not be the cause of the heating.

Some kind of heating is also required to explain the chromosphere common among $\mathrm{M}$ giants, although no UV or $\mathrm{H} \alpha$ observations of BK Vir are available in the literature. However, the (electron) temperature of the chromosphere inferred from the UV emission lines in M giants is much higher ( 6000-10000 K, Eaton \& Johnson 1988; Luttermoser et al. 1994) than the 1900-2100 K derived for the inner CO layer. Therefore, although the inner CO layer is warmer than the uppermost layer of the photosphere, the $\mathrm{CO}$ gas in the extended outer atmosphere represents a distinct component other than the chromosphere.

Still, the process responsible for the chromospheric heating may lead to the formation of the hot plasma and warm molecular gas in the outer atmosphere. For example, the magnetohydrodynamical (MHD) simulation of stellar winds in red giants by Suzuki (2007) shows the formation of transient hot gas bubbles embedded in cool gas outflows, although the simulation is still limited to stars with relatively high effective temperatures $(\gtrsim 3900 \mathrm{~K})$ and much less luminous stars $\left(\$ 1000 L_{\odot}\right)$ compared to BK Vir. However, as Airapetian et al. (2010) point out, this simulation assumes fully-ionized plasma, although the gas around $\mathrm{K}-\mathrm{M}$ giants is only weakly ionized. The MHD simulation of Airapetian et al. (2010) reproduces the observed terminal wind velocities and mass-loss rates of $\mathrm{K}$ giants, but the agreement between the modeled and observed radial velocity fields is not yet satisfactory (a comparison of the model of Airapetian et al. 2010 and the observed data summarized in Crowley et al. 2009 is given in Harper 2011). Since the simulation of Airapetian et al. (2010) assumes a nearly iso-thermal chromosphere, it does not include the cool, molecular component. The extension of these MHD simulations to cooler and more luminous stars including the modeling of thermal structures would be useful for understanding the origin of the MOLsphere in normal red giants.

The presence of the dense $\mathrm{CO}$ layers above the photosphere suggests that there can also be other molecular species in the MOLsphere. For example, TiO is one such molecule, and the contribution from the MOLsphere can significantly affect the strength of the TiO bands observed in the optical. Hron et al. (2010) show that the MOLsphere models for the red supergiant Betelgeuse available in the literature predict the $\mathrm{TiO}$ bands to be too strong compared to the observed ones. This is because of the strong contribution from the $\mathrm{TiO}$ molecules in the MOLsphere. As possible reasons, the authors suggest the clumpy or patchy nature of the MOLsphere with a small filling factor and/or the crude nature of the current MOLsphere models. In addition, we suggest that the $\mathrm{TiO}$ bands may form by scattering in the MOLsphere. The line formation by scattering for electronic transitions (such as the TiO bands) is suggested by Hinkle \& Lambert (1975). The construction of the MOLsphere model with this process taken into account is beyond the scope of this paper, but should be pursued to obtain a more comprehensive picture of the MOLsphere.

\section{Concluding remarks}

We have spatially resolved the normal M7 giant BK Vir in the individual CO first overtone lines with VLTI/AMBER. The uniform-disk diameter shows clear increases by $12-31 \%$ in the CO lines compared to the continuum. We detected non-zero/non$\pi$ CPs and non-zero DPs in the CO lines, which reveal an asymmetry in the upper photosphere and outer atmosphere where the CO lines form. The visibilities, CPs, and DPs observed in the 
individual CO lines are symmetric with respect to the line center. This is in marked contrast with the red supergiant Betelgeuse, in which the vigorous inhomogeneous gas motions manifest themselves as the asymmetry in the visibilities in the blue and red wing of the lines. The absence of this asymmetry indicates that the amplitude of the inhomogeneous velocity field in BK Vir is much smaller $\left(\$ 5 \mathrm{~km} \mathrm{~s}^{-1}\right)$ than in Betelgeuse.

While the MARCS photospheric model can satisfactorily reproduce the observed CO line spectrum of BK Vir, the angular size in the CO lines predicted by the model is too small. This indicates the presence of more extended CO layers than predicted by the photospheric model. Our model with two CO layers added above the MARCS model can explain the observed spectrum, visibilities, and CPs reasonably well. The derived temperatures of the two extended CO layers, 1900-2100 K and 1500-2100 K, are higher than, or equal to, that of the uppermost layer of the photosphere. This result suggests the operation of some heating mechanism in the outer atmosphere within $\sim 3 R_{\star}$.

The present work illustrates that AMBER observations with high-spectral resolution are effective for constraining the physical properties of the outer atmosphere. Given that normal red giants with small variability amplitudes are not yet intensively studied by IR spectro-interferometry, it is indispensable to carry out a systematic survey of a sample of these stars with ranges of temperatures and luminosities. This will shed new light on the mass-loss mechanism not only in normal red giants but also in Mira stars.

Acknowledgements. We thank the ESO VLTI team for supporting our AMBER observations. NSO/Kitt Peak FTS data on the Earth's telluric features were produced by NSF/NOAO. This publication makes use of data products from the Two Micron All Sky Survey, which is a joint project of the University of Massachusetts and the Infrared Processing and Analysis Center/California Institute of Technology, funded by the National Aeronautics and Space Administration and the National Science Foundation.

\section{References}

Airapetian, V. S., Carpenter, K. G., \& Ofman, L. 2010, ApJ, 723, 1210 Bedding, T., Zijlstra, A. A., von der Lühe, O., et al. 1997, MNRAS, 286, 957 Bertelli, G., Girardi, L., Marigo, P., \& Nasi, E. 2008, A\&A, 484, 815 Crowley, C., Espey, B. R., Harper, G. M., \& Roche, J. 2009, Proceedings of the 15th Cambridge Workshop on Cool Stars, Stellar Systems and the Sun, AIP Conf. Proc., 1094, 267

De Beck, E., Decin, L., de Koter, A., et al. 2010, A\&A, 523, 18

Dyck, H. M., van Belle, G. T., \& Thompson, R. R. 1998, AJ, 116, 981

Eaton, J. A. 1995, AJ, 109, 1797

Eaton, J. A., \& Johnson, H. R. 1988, ApJ, 325, 355

Famaey, B., Jorissen, A., Luri, X., et al. 2005, A\&A, 430, 165

Fouque, P., Le Bertre, T., Epchtein, N., Guglielmo, F., \& Kerschbaum, F. 1992, A\&AS, 93, 151

Gontscharov, G. A. 2006, Astron. Lett., 32, 759

González Delgado, D., Olofsson, H., Kerschbaum, F., et al. 2003, A\&A, 411, 123

Goorvitch, D. 1994, ApJS, 95, 535

Gustafsson, B., Edvardsson, B., Eriksson, K., et al. 2008, A\&A, 486, 951

Harper, G. 2011, Presentation at From Atoms to Stars: the impact of Spectroscopy on Astrophysics,

http://media.atomstars.org/presentations/harper.pdf

Herwig, F. 2005, ARA\&A, 43, 435
Hinkle, K. H., \& Lambert, D. L. 1975, MNRAS, 170, 447

Hron, J., Aringer, B., \& Paladini, C. 2010, Poster presentation at The Origin and Fate of the Sun: Evolution of Solar-mass Stars Observed with High Angular Resolution, http://www.eso.org/sci/meetings/2010/ stars2010/Presentations/P-hron_molsphere-poster.pdf

Isabel Pérez Martínez, M., Schröder, K.-P., \& Cuntz, M. 2011, MNRAS, 414, 418

Ita, Y., Tanabé, T., Matsunaga, N., et al. 2007, PASJ, 59, S437

Karovska, M., Hack, W., Raymond, J., \& Guinan, E. 1997, ApJ, 482, L175

Kerschbaum, F., \& Hron, J. 1994, A\&AS, 106, 397

Lebzelter, T., \& Hron, J. 1999, A\&A, 351, 533

Lebzelter, T., Kerschbaum, F., \& Hron, J. 1995, A\&A, 298, 159

Luck, R. E., \& Wepfer, G. G. 1995, AJ, 110, 2425

Luttermoser, D. G., Johnson, H. R., \& Eaton, J. A. 1994, ApJ, 422, 351

Lyubimkov, L. S., Lambert, D. L., Rostopchin, S. I., Rachkovskaya, T. M., \& Poklad, D. B. 2010, MNRAS, 402, 1369

Martí-Vidal, I., Marcaide, J. M., Quirrenbach, A., et al. 2011, A\&A, 529, A115

McMurry, A. D., \& Jordan, C. 2000, MNRAS, 313, 423

Mondal, S., \& Chandrasekhar, T. 2004, MNRAS, 348, 1332

Neugebauer, G., \& Leighton, R. B. 1969, Two-micron sky survey, NASA SP3047, Washington

Ohnaka, K. 2004, A\&A, 424, 1011

Ohnaka, K., Bergeat, J., Driebe, T., et al. 2005, A\&A, 429, 1057

Ohnaka, K., Scholz, M., \& Wood, P. 2006, A\&A, 446, 1119

Ohnaka, K., Hofmann, K.-H., Benisty, M., et al. 2009, A\&A, 503, 183

Ohnaka, K., Weigelt, G., Millour, F., et al. 2011, A\&A, 529, A163

Origlia, L., Rood, R., Fabbri, S., et al. 2007, ApJ, 667, L85

Origlia, L., Rood, R., Fabbri, S., et al. 2010, ApJ, 718, 522

Perrin, G. 2003, A\&A, 400, 1173

Perrin, G., Coudé du Foresto, V., Ridgway, S. T., et al. 1998, A\&A, 331, 619

Perrin, G., Ridgway, S. T., Coudé du Foresto, V., et al. 2004, A\&A, 418, 675

Petrov, R. G., Malbet, F., Weigelt, G., et al. 2007, A\&A, 464, 1

Pluzhnik, E., Ragland, S., le Coroller, E., et al. 2009, ApJ, 700, 114

Ragland, S., Traub, W. A., Berger, J.-P., et al. 2006, ApJ, 652, 650

Ragland, S., le Coroller, H., Pluzhnik, E., et al. 2008, ApJ, 679, 746

Richichi, A., \& Percheron, I. 2005, A\&A, 434, 1201

Richter, He., Wood, P. R., Woitke, P., Bolick, U., \& Sedlmayr, E. 2003, A\&A, 400, 319

Samus, N. N., Durlevich, O. V., et al. 2009, General Catalogue of Variable Stars, GCVS database, Version 2011 Jan., Institute of Astronomy of Russian Academy of Sciences and Sternberg State Astronomical Institute of the Moscow State University, Moscow

Schlegel, D., Finkbeiner, D., \& Davis, M. 1998, ApJ, 500, 525

Skrutskie, M. F., Cutri, R. M., Stiening, R., et al. 2006, AJ, 131, 1163 (The 2MASS All-Sky Catalog of Point Sources)

Suzuki, T. K. 2007, ApJ, 659, 1592

Takami, H., Goto, M., Gaessler, W., et al. 2009, PASJ, 61, 623

Takeda, Y., Sato, B., \& Murata, D. 2008, PASJ, 60, 781

Tatulli, E., Millour, F., Chelli, A., et al. 2007, A\&A, 464, 29

Tsuji, T. 2001, A\&A, 376, L1

Tsuji, T. 2008, A\&A, 489, 1271

Tsuji, T., Ohnaka, K., Aoki, W., \& Yamamura, I. 1997, A\&A, 320, L1

Tuthill, P. G., Haniff, C. A., \& Baldwin, J. E. 1999, MNRAS, 306, 353

van Leeuwen, F. 2007, A\&A, 474, 653

Wallace, L., \& Hinkle, K. H. 1996, ApJS, 107, 312

Weigelt, G., Balega, Y., Hofmann, K.-H., \& Scholz, M. 1996, A\&A, 316, L21

Wiedemann, G., Ayres, T. R., Jennings, D. E., \& Saar, S. H. 1994, ApJ, 423, 806

Winters, J. M., Le Bertre, T., Jeong, K. S., Nyman, L.-Å., \& Epchtein, N. 2003, A\&A, 409, 715

Wittkowski, M., Boboltz, D. A., Ohnaka, K., Driebe, T., \& Scholz, M. 2007, A\&A, 470, 191

Wittkowski, M., Boboltz, D. A., Driebe, T., et al. 2008, A\&A, 479, L21

Wittkowski, M., Boboltz, D. A., Ireland, M., et al. 2011, A\&A, 532, L7

Woodruff, H. C., Ireland, M. J., Tuthill, P. G., et al. 2009, ApJ, 691, 1328

Zacharias, N., Monet, D. G., Levine, S. E., et al. 2004, BAAS, 36, 1418, Naval Observatory Merged Astrometric Dataset (NOMAD) 